m37.p05

\title{
Small-angle scattering from three phase systems: application to a coal undergoing an extraction process
}

\section{$\underline{\text { S. Ciccariello }}^{\mathrm{a}}$, P. Riello ${ }^{\mathrm{b}}$}

\author{
aDep.t of Physics "G. Galilei" University of Padova.

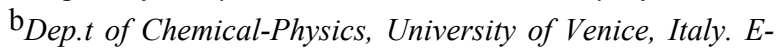 \\ mail: ciccariello@pd.infn.it
}

Keywords: small-angle scattering, multiphase materials,
coal

Metallic catalysts form a classical example of three phase samples with a phase made up of pores. Filling up the pores with a gas at different pressures, the scattering density of the pores will assume different values depending on the considered pressure, while the scattering densities of the other two phases, the support and the metallic particles, remain unchanged and the same happens for the geometrical configurations of the three phases. This last property implies that: a) the so-called stick probability functions of the three phases do not change, b) the correlation function of the full sample is a linear combination of these three functions and c) the scattering intensity is the same linear combination of the Fourier transforms of the same three functions. In this way, after collecting the scattering intensities at three or more different pressure of the gas filling the porous phase, one has enough information to determine the stick probability functions of each constituting phases. Clearly, this technique generalizes the "contrast matching" or the "pore-masquant" techniques. We illustrate an application of this techniques to the case of a coal that underwent fluid extraction processes at different pressures/temperatures. 